\title{
Technology maturity mapping and evolutionary potential forecast of cotton picker based on TRIZ evolution theory
}

\author{
Jianhua SUN, Changqing GAO*, Yuansong ZHENG, Shuxue YIN \\ School of Mechanical Engineering, University of Jinan, Jinan 250022, China \\ a695662900@qq.com, bme_gaocq@ujn.edu.cn, 1248995968@qq.com, d1563121915@qq.com
}

\begin{abstract}
Keywords: TRIZ; Technology forecasting; Technology maturity; Evolutionary potential
Abstract. Technology forecasting is helpful for enterprises to achieve innovation at a high rate of speed. Forecasting technology maturity and evolution potential of products, developing proper strategies and locking technology development direction in the future are important contents of technology forecasting. This paper uses TRIZ technology forecasting method and researches relevant patent information in the analysis process of cotton picker technology. By comparing fitting curve and standard curve and drawing evolutionary potential radar map, we forecast the technology maturity and potential evolution direction of cotton picker. The conclusion proved that TRIZ technology forecasting methodology has a great significance in improving success rates of product innovation and shortening the invention circle.
\end{abstract}

\section{Introduction}

With the technological development and economic growth, how to improve the success rate of innovation to the greatest extent and reduce the cycle of the invention become the key to win our competition $^{[1]}$. Technology forecasting is a process that technological innovation subjects project the technology direction in the future, choose potential development route and speed up the innovation on the basis of fully mastering all kinds of information and using the scientific method according to their own development goals ${ }^{[2]}$.

Any prediction research to succeed depends on whether the researchers find the past development rule and get the information and data which influence and determine the future development trend. The statistics of the World Intellectual Property Organization shows patents contained 90\%-95\% technology information in the world. Compared to other information resources, patent documents have many characteristics, such as original in contents, format specifications and scientific classification $^{[3,4]}$. Therefore, we choose patent literature as the basis data of technology forecasting.

\section{Technology Maturity Mapping.}

Technology maturity mapping is a method to predict the evolution degree of technology system. The purpose is to speculate which stage the current technology stay in the technology lifecycle, and assist enterprises to make appropriate development strategy according to the characteristics of different stages.

Patent Characteristic Curve. Altshuller inspected how the product performance, number of patents, level of patents and product profit are changing in time through analyzing nearly 1.25 million pieces of high level invention patent. And he draw the patent characteristic curve that embodied the evolution laws of technology system, as shown in Fig. 1.

It is difficult to obtain patent performance and product profit in practical analysis, therefore Darrell Mann proposed two kinds of special patents: symptom curing patents and cost reduction related patents. And he draw the curves how these two parameters are changing in time, as shown in Fig. 2.

$\mathbf{S}$ curve and $\mathbf{S}$ curves. The evolution of technical system characters with "S" sharp, and runs through the four stages of S curve: infancy stage, growth stage, mature period, decline phase, as shown in Fig. 3. S curve completely described the life cycle of a technical system ${ }^{[5]}$. 


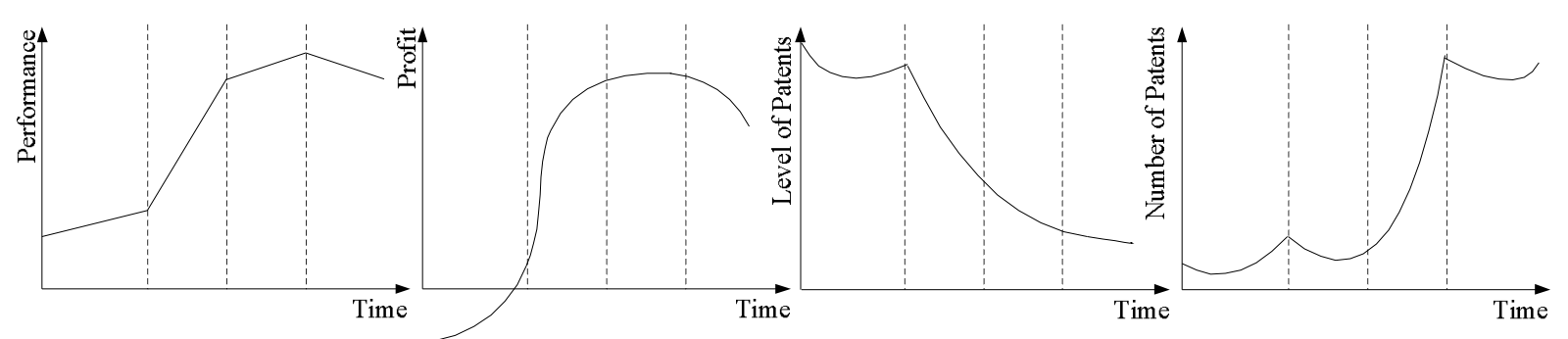

Fig. 1 Patent Characteristic Curve

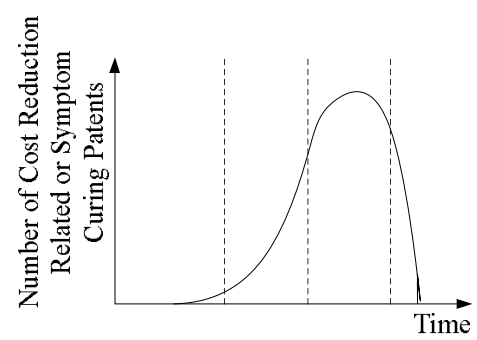

Fig. 2 Characteristic Curve of Special Patents

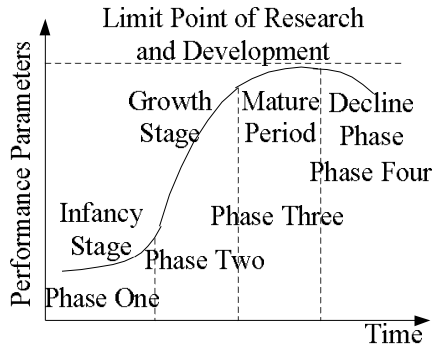

Fig. 3 Four Stages of Curve Development

When technical system evolves to a certain degree, reaches development point and enters decline phase, inevitably, there will be a new technical system to replace it. Thus existing technology replaces the old technology, and new technology replaces the existing technology, over and over again, $\mathrm{S}$ curves formed.

\section{Technology evolutionary potential forecasting.}

Specific to the evolutional status and evolutionary potential of each technical system, it is need to choose evolutionary route under the guidance of evolution model to forecast evolutionary potential.

Technological evolution model. Several domestic and overseas TRIZ gurus put forward different evolution models on the basis of Altshuller's traditional evolution theory. These theories include Zusman's ${ }^{[6]}$ directed evolution and guided technology evolution proposed by Fey and Rivin ${ }^{[7]}$. In this paper, we use Altshuller's eight evolution laws in the research process. These laws include: complete technical system, the path of energy transfer, concertedly evolving, alternating dynamic, unbalanced evolution of subsystem, evolve to super-system, evolve to microcosmic and field application added, improve idealized degree.

Technology evolutionary potential radar map. Technical systems evolve to different degree along the different evolution models, and we use technology evolution potential radar map to forecast the level of evolution. Each ray represents an evolution route. Use Numbers to quantify evolution routes and current states of the technical systems, and then link these dots representing current evolution statue in different evolutionary lines. In the diagram, the shadow zone means evolution process that technical systems have finished, and the clear area represents potential evolution fields. The bigger the clear area, the greater the evolutionary potential of technical system.

\section{Application instance.}

Cotton picker is a cotton harvesting machinery, which takes the place of manpower to pick cotton. The main ways of picking cotton are air suction, comb tooth, belt type and spindle type, among them, spindle is the most important and studied picking cotton way. Next, we will proceed technology maturity mapping aimed at spindle picking technology, and forecast evolutionary potential of cotton picker technical system.

Inputting the keyword "cotton picker" in Chinese patent announcement, we retrieved 38 authorized patents for inventions and 285 utility model patents from 1995 to 2016 . After getting rid of 9 irrelevant patents for inventions and 35 irrelevant utility model patents according to IPC, and merging 21 
repeated patents between patents for inventions and utility model patents, finally we got 258 valid patents.

Maturity mapping of spindle-type technology. In this paper, in order to proceed technology maturity mapping, we extracted 102 valid patents applying the principle of spindle from 1997 to 2016. These patents' application number, patent level and the number of symptom curing patents are as shown in Table 1.

Table 1 Statistics on Number and Level of Patents

\begin{tabular}{cccc}
\hline Year & Number of Patents & Level of Patents & Number of Symptom Curing Patents \\
\hline $1997 \sim 1998$ & 1 & 2.2 & 0 \\
$1999 \sim 2000$ & 2 & 2 & 0 \\
$2001 \sim 2002$ & 3 & 2 & 1 \\
$2003 \sim 2004$ & 5 & 1.8 & 2 \\
$2005 \sim 2006$ & 4 & 2 & 1 \\
$2007 \sim 2008$ & 13 & 1.77 & 3 \\
$2009 \sim 2010$ & 16 & 1.63 & 6 \\
$2011 \sim 2012$ & 19 & 1.38 & 9 \\
$2013 \sim 2014$ & 33 & 1.36 & 14 \\
$2015 \sim 2016$ & 6 & 1.33 & 6 \\
\hline
\end{tabular}

Then, we analyze the data in Table 1 and draw patent characteristic curve, as shown in Fig. 4. Compared with the standard curves, we can draw a conclusion that spindle cotton picking technology has entered decline phase.

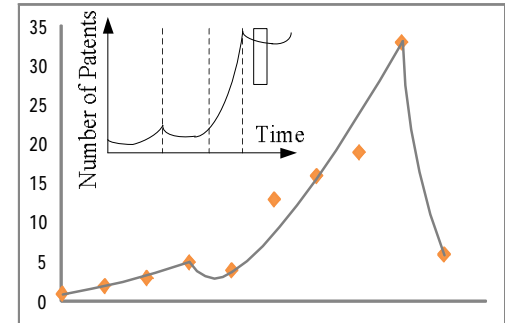

(a)Number of Patents

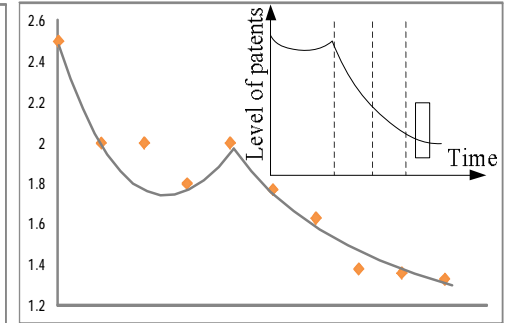

(b)Level of Patents

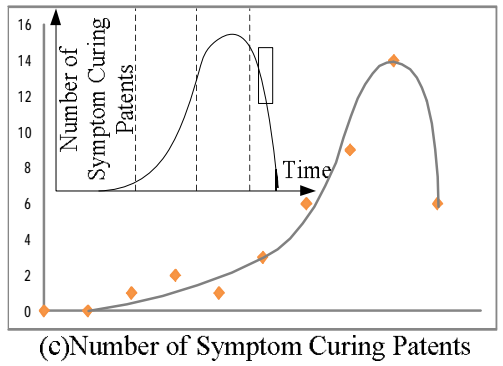

(c)Number of Symptom Curing Patents

Fig. 4 Characteristic and Standard Curves of Spindle type Cotton-picking

Evolutionary potential forecasting of cotton picker. We select 7 evolution route on the basis of analysis of 258 valid patents related cotton picker. And then quantify the evolution degree, 0 means that technology begins to evolve along a certain evolution route, the greater the number, the higher the evolution model. In the end, sequentially link all the dots representing current evolution status, we got the technology evolutionary potential radar map of cotton picker, as shown in Fig. 5.

Evolution route 1: Reduce human invention

Human - Human + Tools $\rightarrow$ Human + Semi-automatic tools $\rightarrow$ Human + Automatic tools $\rightarrow$ Automatic tools

Evolution route 2: Increase freedom

1 freedom $\rightarrow 2$ freedoms $\rightarrow 3$ freedoms $\rightarrow$ Many freedoms

Evolution route 3: Controllability

Direct control $\rightarrow$ Indirect control $\rightarrow$ Feedback control $\rightarrow$ Intelligent control

Evolution route 4: Remove impurities

Manual pick $\rightarrow$ Mechanical separation $\rightarrow$ Chemical separation $\rightarrow$ Optical separation $\rightarrow$ Self repel

Evolution route 5: From single to multiple

Single system Dual system $\rightarrow$ Three-system $\longrightarrow$ Multisystem


Evolution route 6: Functional grouping

Monofunctional body Major function + Additional function Two functions Three functions

Evolution route 7: Simplify subsystem

Simplify transmission system Simplify power system Simplify control system $\rightarrow$ Simplify execution system

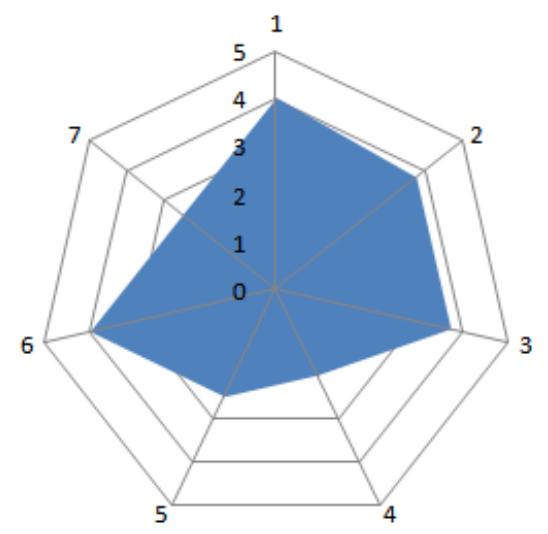

Fig. 5 Technology Evolutionary Potential Radar Map of cotton picker

\section{Conclusions}

(1)The above technology maturity prediction result shows that spindle type cotton-picking technology has entered a recession since 2015, so the development of cotton-picking technology should go into a new $\mathrm{S}$ curve, namely enterprises should further develop alternative technologies to optimize product performance.

(2)The result of technology evolutionary potential forecasting shows that cotton picker technologies still have great evolutionary potential on the aspect of cotton impurities removing. Actual situation also confirmed that the development of impurities removing subsystem is slower than other subsystems. The evolution route of impurities removing offers us a researchable evolution direction.

\section{Acknowledgements}

This work was financially supported by Science and Technology Development Plan Project of Shandong Province (2014GGX106003), Large Equipment Upgrade Project of Shandong Province (2012SJGZ15).

\section{References}

[1] Wenfeng ZHANG, in: Research of Product Innovation Design Based on QFD. Zhejiang University,(2008). In Chinese.

[2] Yunjun ZHANG: submitted to Journal of Intelligence(2014). In Chinese.

[3] Information on http//:www.European-patent office.org/espacenet/info/ index.Htm.

[4] Xianghui LV, Baoyan QIU and Hong QIAO: submitted to Computer and Information Technology(2008). In Chinese.

[5] Min ZHAO, Wucheng ZHANG and Guanzhu WANG, in: TRIZ Enhancement and Practical Application, China Machine Press(2015). In Chinese.

[6] Zusman A, Zlotin B and Zainiev G, in: Directed Evolution : Philosophy, Theory and Practice, Ideation International( 2002).

[7] Fey V R, Rivin E I: submitted to TRIZ Journal(1991). 